\title{
The clinical utility of ANCA positivity
}

It is six years since a leader in the Annals reviewed the performance of antineutrophil cytoplasmic antibodies (ANCA) in clinical practice. ${ }^{1}$ At that time there was increasing confidence in the value of ANCA testing in the diagnosis of Wegener's granulomatosis and related primary vasculitides. Sensitivity of cANCA for Wegener's granulomatosis was reported as $50 \%-96 \%$ and specificity was $90 \%-100 \%$. What is our current understanding of the place of ANCA testing in the diagnostic process?

\begin{abstract}
Methodology
Clinicians need to be aware of the different methods used to detect ANCA, their relative merits, and their potential pitfalls. Indirect immunofluorescent examination of a patient's serum on ethanol fixed neutrophils remains the method of choice. ${ }^{2}$ Two major staining patterns, classic cANCA and perinuclear pANCA, can be identified; however, the recognition of the distinct patterns is not always easy and particular care is needed to ensure that ANCA are not confused with other autoantibodies and vice versa. ${ }^{3}$ The main target antigens of ANCA are proteinase 3 (cANCA) and myeloperoxidase (pANCA) but antibodies to several other antigens will produce the patterns of pANCA (lactoferrin, cathepsin G, and elastase) and cANCA (cationic protein 57). Despite this, the correlation of indirect immunofluorescence pattern with antigen is not absolute, and the clinical importance of the different antigenic specificities of ANCA is not entirely established. ${ }^{45}$ Antigen specific, solid phase assays have been developed to provide quantitative results, but multiple assays would be required to completely replace indirect immunofluorescence and these are not yet standardised. ${ }^{6}$ A reliable ANCA service continues, therefore, to be dependent on the accurate interpretation of findings from indirect immunofluorescence.
\end{abstract}

\section{Disease associations}

Davies et al first described ANCA in a series of eight patients with necrotising glomerulonephritis in whom there was serological evidence of recent arbovirus infection. ${ }^{7}$ In 1985 , van der Woude et al proposed that ANCA were useful both in the diagnosis and monitoring of disease activity in Wegener's granulomatosis. ${ }^{8}$ As the indirect immunofluorescence patterns were defined, it seemed that cANCA was associated almost exclusively with Wegener's granulomatosis and pANCA with renal limited forms of microscopic polyangiitis. ${ }^{9}$ A further, intermediate staining pattern termed atypical or xANCA was described in ulcerative colitis. ${ }^{10}$ Isotype specificity was also reported. IgA ANCA were described in HenochSchonlein purpura and IgM ANCA were reported in association with severe pulmonary haemorrhage. ${ }^{11} \mathrm{~A}$ distinct indirect immunofluorescence pattern was described in Kawasaki disease, although this has recently been retracted by that group. ${ }^{12}$ It also became clear that ANCA occurred in a wide range of other clinical conditions including infection, connective tissue disorders, inflammatory bowel disease, autoimmune hepatitis, and malignancy. ${ }^{13-19}$ These findings identified potentially important causes of "false positive" ANCA results.

\section{Diagnostic value}

Establishing the diagnostic value of any new test is dependent on having an existing clinical and pathological "gold standard" and this has been a particular problem in the systemic vasculitides. Initial studies which reported a high sensitivity and specificity of cANCA were based on well defined cases of Wegener's granulomatosis established by biopsy. Those results suggested that ANCA were helpful in the classification of already diagnosed patients but did not entirely establish the diagnostic value of the test. Positive predictive value is the most useful characteristic in understanding what a positive ANCA result means in routine clinical practice. Positive predictive value, like sensitivity and specificity, is not an absolute characteristic, however, and is critically influenced by prevalence of disease. ${ }^{20}$ As a result, preselection of patient populations will significantly affect the apparent positive predictive value obtained.

Three groups have recently attempted to establish a positive predictive value for ANCA, each attempting to avoid preselection bias and each with different diagnostic gold standards. ${ }^{21-23}$ Davenport et al reviewed 738 of 779 (95\%) consecutive requests for ANCA detection received by their regional immunology laboratory. ${ }^{21}$ Their diagnostic criteria included a strict requirement for histological confirmation of Wegener's granulomatosis or microscopic polyangiitis. Positive ANCA results were obtained for 183 of 738 serum samples $(25 \%)$. The overall prevalence of Wegener's granulomatosis, microscopic polyangiitis, and the syndrome of systemic vasculitis was $17 \%$ and they showed a positive predictive value of $40 \%$ for this group of three related conditions.

Our group reviewed 301 of $327(92 \%)$ consecutive positive ANCA tests detected by a different regional immunology laboratory. ${ }^{22}$ The diagnostic criteria used were less strict than those of Davenport et $a l^{21}$ in that clinical diagnoses were accepted after critical chart review. The positive predictive value for a group of primary vasculitic disorders was $26.9 \%$ (cANCA $38 \%$ and pANCA $20 \%$ ). However, a number of other factors were identified which had important effects on the positive predictive value of ANCA. These included pattern, titre, confirmation of pANCA as anti-myeloperoxidase specific, and whether or not there was an associated antinuclear antibody. The positive predictive value of both pANCA and cANCA increased as the ANCA titre increased but this did not happen if there was an associated antinuclear antibody in the patient's serum. Confirmation of pANCA as antimyeloperoxidase specific by solid phase assay improved the positive predictive value from $20 \%$ to $66 \%$. The positive predictive value for high titre cANCA, in the absence of antinuclear antibody, was $90 \%$.

Rao et al have prospectively examined the value of cANCA in the diagnosis of Wegener's granulomatosis by studying 346 consecutive patients "suspected of having vasculitis". ${ }^{23}$ They used the 1990 American College of Rheumatology (ACR) clinical classification criteria to diagnose Wegener's granulomatosis but did not require histological confirmation. ${ }^{24}$ They reviewed 212 of 252 $(84 \%)$ eligible patients, 25 of whom fulfilled the ACR definition and found positive predictive values of $50 \%$ for diagnosis by ACR criteria, 36\% for disease established by 
biopsy. Unfortunately 19 of those initially diagnosed with Wegener's granulomatosis were subsequently classified as having another diagnosis during follow up, questioning the applicability of ACR criteria in this study.

\section{Prognostic value}

In early reports it was noted that patients with active Wegener's granulomatosis tended to have higher titres of ANCA than those with inactive disease. ${ }^{8}$ In a prospective study of 58 patients with Wegener's granulomatosis established by biopsy, less immunosuppressive therapy was required if treatment was guided by a rise in ANCA titre rather than waiting for exacerbation of disease. ${ }^{25}$ Different groups have also found ANCA titres of value in predicting disease relapse. ${ }^{26}{ }^{27}$ However, other data indicate continuing uncertainty regarding this use of ANCA testing even in well defined patient groups. ${ }^{28}{ }^{29}$ In particular, the Bristol group found a significant risk of treating intercurrent infection inappropriately with immunosuppressive therapy when evaluating this strategy. ${ }^{28}$

Is there prognostic significance when ANCA are detected in patients with connective tissue disorders? In 40 serum samples from patients with systemic lupus erythematosus, Schnabel et al found no correlation between detection of ANCA and organ involvement (as defined by the British Isles Lupus Assessment Group index) or with the occurrence of vasculitis. ${ }^{17}$ Whereas it was suggested that ANCA did not occur in uncomplicated rheumatoid arthritis but only in Felty's syndrome, there is evidence that ANCA are present in a subset of patients with rheumatoid arthritis who are seropositive, have extraarticular disease, and require immunosuppressive therapy more often than expected. ${ }^{15} 162230$ This does not, however, lead to the conclusion that ANCA testing should be used for the identification of such patients.

\section{ANCA in pathogenesis}

Autoantibodies are classified as primary or secondary depending on whether they are recognised as having a primary role in pathogenesis of disease. The history of autoimmune serology shows that examples of primary autoantibodies are rare but examples include antiacetyl choline receptor antibodies in myasthenia gravis and antiglomerular basement membrane antibodies in Goodpasture's syndrome. Secondary autoantibodies are much more common and despite their lack of a clearly defined role in causation of disease, they have a valuable role in diagnosis and classification of clinical conditions.

The initial studies which suggested a highly specific association between ANCA and the occurrence and activity of vasculitic disorders raised expectations that ANCA might be primarily involved in pathogenesis. Early in vitro work suggested a model whereby ANCA might activate neutrophils in an antigen specific manner and cause endothelial damage. ${ }^{31} 32$ More recently, it has been shown that such activation is not necessarily antigen specific. ${ }^{33}{ }^{34}$ A further major difficulty has been the failure to establish an appropriate animal model based on the effect of ANCA alone. There is, therefore, interest in alternative models of disease including the influences of idiotypic dysregulation and the contribution of T cells. ${ }^{35} 36$

Confirming the autoimmune nature of a disease and establishing a primary role for autoantibodies requires a combination of circumstantial, indirect, and direct evidence. ${ }^{37}$ Although there continues to be widespread interest and research effort in establishing an autoimmune basis for ANCA associated vasculitis, direct proof is still lacking.
The current laboratory findings and many of the emerging clinical characteristics of ANCA would be consistent with a secondary autoantibody occurring in the context of an incompletely understood pathological process.

\section{Conclusions}

Detection of ANCA requires expertise which is usually only available in specialist immunology laboratories. Indirect immunofluorescence remains the method of choice and although there is evidence that myeloperoxidase specific assays are helpful for interpretation of pANCA, solid phase assays are currently an unproved, expensive alternative to indirect immunofluorescence. To further clarify the predictive value of ANCA, prospective clinical studies are needed in which agreed minimum diagnostic criteria are used. These criteria must exclude ANCA serology; the inclusion of biopsy findings at least consistent with the diagnosis would seem appropriate. The place of ANCA in pathogenesis of disease remains an unanswered question. It should be remembered, however, that secondary autoantibodies can prove extremely valuable in clinical practice.

ANCA testing should remain a part of the investigation of patients in whom Wegener's granulomatosis and related vasculitides are suspected, but it cannot replace careful clinical assessment and the appropriate use of other investigations including serology, radiology, biochemistry, and histology. A high titre cANCA, detected in a patient with appropriate clinical features, is supportive evidence for Wegener's granulomatosis or a closely related vasculitis; however, the absence of ANCA does not exclude these diseases. By contrast, a positive ANCA result without other clinical evidence for Wegener's granulomatosis, may represent a false positive and a wide differential diagnosis must include the other potentially treatable diseases already mentioned. With regard to monitoring patients with Wegener's granulomatosis, ANCA titres should be considered as part of a careful clinical and laboratory evaluation of disease activity. In some cases ANCA titres will be of value, but not in all. There is no evidence to justify the use of ANCA testing in monitoring the activity of other diseases, including rheumatoid arthritis.

At present the evidence suggests that less than half of all ANCA detected are truly indicative of a vasculitic disorder. The ANCA test is, therefore, not suitable as a screening test for vasculitis and there is an appreciable risk associated with overreliance on an ANCA result (positive or negative). The test is not uniformly positive in patients with vasculitic disorders but may be positive in a wide range of other clinical conditions. Many of those nonvasculitic, ANCA associated diseases could be exacerbated by inappropriate immunosuppressive therapy. Despite these cautions, ANCA testing still has a potentially valuable role in this difficult area of diagnosis. As with many other tests, the predictive value of the result is proportional to the clinical likelihood of the disease in question.

Belfast City and Royal Victoria Hospitals,

Belfast,

J D M EDGAR

Northern Ireland

\section{Correspondence to: Dr Edgar}

1 Ramirez G, Khamashta M A, Hughes G R V. The ANCA test: its clinical relevance. Ann Rheum Dis 1990; 49: 741-2.

2 Wiik A. Delineation of a standard procedure for indirect immunofluorescent detection of ANCA. Acta Pathologica Microbiologica Immunologica

3 Spickett G P, Broomhead V. Formalin fixation and patterns of antineutrophil cytoplasmic antibodies. F Clin Pathol 1995; 48: 89-90.

Segelmark M, Baslund B, Wieslander J. Some patients with antimyeloperoxidase autoantibodies have a c-ANCA pattern. Clin Exp Imyeloperoxidase autoantibod $1994 ; 96: 458-65$. 
5 Baslund B, Segelmark M, Wiik A, Szpirt W, Peterson J, Wieslander J. Screening for anti-neutrophil cytoplasmic antibodies (ANCA): is indirect immunofluorescence the method of choice? Clin Exp Immunol 1995; 99. 486-92.

6 Hagen E C. Development and standardisation of solid-phase assays for the detection of anti-neutrophil cytoplasmic antibodies (ANCA) for clinical application: report of a large clinical evaluation study. Clin Exp Immunol 1995; 101 (suppl 1): 29 .

7 Davies D J, Moran J E, Niall J F, Ryan G B. Segmental necrotising glomerulonephritis with antineutrophil antibody: possible arbovirus glomerulonephritis with antin

8 van der Woude F J, Rasmussen N, Lobatto S, et al. Autoantibodies against neutrophils and monocytes: tool for diagnosis and marker of disease activity in Wegener's Granulomatosis. Lancet 1985; 1: 425-9.

9 Jenette J C, Falk R J. Antineutrophil cytoplasmic auto-antibodies and associated diseases: a review. Am $\mathcal{F}$ Kidney Dis 1990; 15: 517-29.

10 Rump J A, Scholmerich J, Gross V, et al. A new type of perinuclear antineutrophil cytoplasmic antibody (p-ANCA) in active ulcerative colitis but not in Crohn's disease. Immunobiology 1990; 181: 406-13.

11 Esnault V L M, Ronda N, Jayne D R W, Lockwood C M. Association of ANCA isotype and affinity with disease expression. F Autoimmun 1993; 6: $197-205$.

12 Nash M C, Shah V, Reader J A, Dillon M J. Anti-neutrophil cytoplasmic antibodies and anti-endothelial cell antibodies are not increased in Kawasaki disease. $B r \mathcal{F}$ Rheumatol 1995; 34: 882-7.

13 Mains B. Wegener's granulomatosis: false positive anti-neutrophil cytoplasmic antibody test. $\mathcal{F}$ Laryngol Otol 1989; 103: 524-5.

14 Efthimou J, Spickett G, Lane D, Thompson A. Antineutrophil cytoplasmic antibodies, cystic fibrosis, and infection [letter]. Lancet 1991; 337: 1037-8.

15 Juby C, Johnston C, Davis P, Russell A S. Antinuclear and antineutrophil cytoplasmic antibodies (ANCA) in the sera of patients with Felty's syndrome. Br $\mathcal{F}$ Rheumatol 1992; 31: 185-8.

16 Coremans I E M, Hagen E C, Daha M R, et al. Antilactoferrin antibodies in patients with arthritis are associated with vasculitis. Arthritis Rheum 1992; 35: 1466-75.

17 Schnabel A, Csernok E, Isenberg D A, Mrowka C, Gross W L Antineutrophil cytoplasmic antibodies in systemic lupus erythematosus: Antineutrophil cytoplasmic antibodies in systemic lupus erythematosus: prevalence, $633-7$.

18 Targan S R, Landers C, Vidrich A, Czaja A J. High-titer antineutrophil cytoplasmic antibodies in type-1 autoimmune hepatitis. Gastroenterolog 995; 108: 1159-66.

19 Edgar J D M, Rooney D P, McNamee P, McNeill T A. An association between ANCA positive renal disease and malignancy. Clin Nephrol 1993 40: $22-5$.

20 Vecchio T J. Predictive value of a single diagnostic test in unselected populations. Lancet 1966; 274: 1171-3.
21 Davenport A, Lock R J, Wallington T B, Feest T G. Clinical significance of anti-neutrophil cytoplasm antibodies detected by a standardised indirect immunofluorescence assay. $Q \mathcal{F} M e d$ 1994; 87: 291-9.

22 Edgar J D M, McMillan S A, Bruce I, Conlan S K. An audit of ANCA in routine clinical practice. Postgrad Med f 1995; 71: 605-12.

23 Rao J K, Allen N B, Feussner J R, Weinberger M. A prospective study of antineutrophil cytoplasmic antibody (cANCA) and clinical criteria in diagnosing Wegener's granulomatosis. Lancet 1995; 346: 926-31.

24 Leavitt R Y, Fauci A S, Bloch D A, et al. The American College of Rheumatology 1990 criteria for the classification of Wegener's Rheumatology 1990 criteria for the classificat

25 Tervaert J W C, Huitema M G, Hene R J, et al. Prevention of relapses in Wegener's granulomatosis by treatment based on antineutrophil cytoplasmic antibody titre. Lancet 1990; 336: 709-11.

26 Egner W, Chapel H M. Titration of antibodies against neutrophil cytoplasmic antigens is useful in monitoring disease activity in systemic vasculitides. Clin Exp Immunol 1990; 82: 244-9.

27 Jayne D R W, Gaskin G, Pusey C D, Lockwood C M. ANCA and predicting relapse in systemic vasculitis. $Q \mathcal{F}$ Med $1995 ; 88: 127-33$.

28 Davenport A, Lock R J, Wallington T. Clinical significance of the seria measurement of autoantibodies to neutrophil cytoplasm using a standard indirect immunofluorescence test. Am 7 Nephrol 1995; 15: 201-7.

29 Kerr G S, Fleisher T A, Hallahan C W, et al. Limited prognostic value of changes in anti-neutrophil cytoplasmic antibody titer in patients with changes in anti-neutrophil cytoplasmic antibody titer in patic

30 Braun M G, Csernok E, Schmitt W, Hauschild S, Gross W L. Antineutrophil cytoplasmic antibodies (ANCA) and extra-articular involvement in rheumatoid arthritis (RA) [abstract]. Clin Exp Immuno 993; 93 (suppl): 33.

31 Falk R J, Terrell R S, Charles L A, Jennette J C. Anti-neutrophil cytoplasmic autoantibodies induce neutrophils to degranulate and produce oxygen radicals in vitro. Proc Natl Acad Sci 1990; 87: 4115-9.

32 Gross W L, Schmitt W H, Csernok E. ANCA and associated diseases: mmunodiagnostic and pathogenetic aspects. Clin Exp Immunol 1993; 91 $1-12$.

33 Porges A J, Redecha P B, Kimberly W T, Csernok E, Gross W L, Kimberly R P. Anti-neutrophil cytoplasmic antibodies engage and activate human neutrophils via FcTRIIa. $¥$ Immunol 1994; 153: 1271-80.

34 Mulder A H L, Horst G, Limburg P C, Kallenberg C G M. Activation of granulocytes by anti-neutrophil cytoplasmic antibodies is FcR-dependent. granulocytes by anti-neutrophil cyto

35 Blank $M$, Tomer $Y$, Stein $M$, et al. Immunization with anti-neutrophil cytoplasmic antibody (ANCA) induces the production of mouse ANCA and perivascular lymphocyte infiltration. Clin Exp Immunol 1995; 102: 120-30

36 Luqmani R A, Bacon P A, Beaman M, et al. Classical versus non-rena Wegener's granulomatosis. $Q \mathcal{M}$ Med 1994; 84: 161-7.

37 Rose N R, Bona C. Defining criteria for autoimmune diseases (Witebsky's postulates revisited). Immunol Today 1993; 14: 426-30. 